\title{
Fermilab faces life after the Tevatron
}

\section{As collider shuts down, US particle physicists shift focus.}

\section{BY EUGENIE SAMUEL REICH}

$\mathrm{L}$ ike an old and celebrated race track, the giant particle accelerator known as the Tevatron is down to its final laps.

Shortly after 2 p.m. on 30 September, with reporters watching by video link from a nearby auditorium, an operator at the Fermi National Accelerator Laboratory (Fermilab) in Batavia, Illinois, will divert the final bunches of protons and antiprotons speeding around the Tevatron's 6.3-kilometre ring, sending them barrelling into a solid metal block. The experiment that ruled high-energy physics for more than 25 years will then be over, its funding expired.

The shutdown will provide an occasion to dwell on the Tevatron's past successes (see 'Smashing success'), but it also marks Fermilab's transition to smaller and lower-profile experiments that explore different kinds of physics. Champagne corks are not expected to pop following the closure. "We're not that happy," says Roger Dixon, head of the lab's accelerator division. "It's a solemn occasion."

The closure is a consequence of tight US physics funding and the advent of the Large Hadron Collider (LHC) at CERN, Europe's high-energy physics lab near Geneva, Switzerland. The LHC broke the Tevatron's record for collision energy in 2009 and has been running steadily since 2010. In 2008, a US Department of Energy advisory panel recommended that the country switch its focus in accelerator physics from the 'energy frontier' that would be dominated by the LHC to the 'intensity frontier', in which researchers aim to increase the number of particles produced per second. Instead of creating previously unknown particles by pushing collisions to higher energies, the new strategy will be to investigate rare interactions involving known particles. The lab is already running three experiments to create and study neutrinos - nearly massless particles that interact only weakly with ordinary matter - using protons from accelerators that also feed the Tevatron. By modifying storage rings and accelerators that will become available when the Tevatron closes, Fermilab should be able to bring two more neutrino experiments online by 2014 , followed by two experiments on muons, heavier cousins of the electron. The lab is also working on a proposal for an experiment called Project X, which would increase the intensity of the accelerator beams from 750 kilowatts to more than 2 megawatts, and would allow detailed comparisons of the properties of matter and antimatter. The project has yet to receive approval from the Department of Energy.

For US-based particle physicists, the change is likely to mean smaller research groups perhaps 100 or 200 per experiment, rather than 600 for each of the Tevatron's two detector collaborations. "It's a big shift," says Regina Rameika, project manager of one of the neutrino experiments now under construction.

As for the Tevatron, its detectors and magnets are ultimately destined for the waste dump, but immediate plans are to run tours of the soon-to-be-accessible beam cavities and detectors for some of the roughly 15,000 school children who visit Fermilab each year. Meanwhile, scientists working at the Tevatron will continue to analyse the data that it has produced - looking for signals of the Higgs particle that is thought to endow all others with mass, or for evidence that will narrow the probable range of its mass. "We expect we will be adding to our legacy," says Dmitri Denisov, spokesman for the Tevatron's DZero collaboration.

\section{SMASHING SUCCESS}

Discoveries at the Tevatron particle collider (pictured, below) confirmed the standard model of physics and set the course for new investigations beyond it.

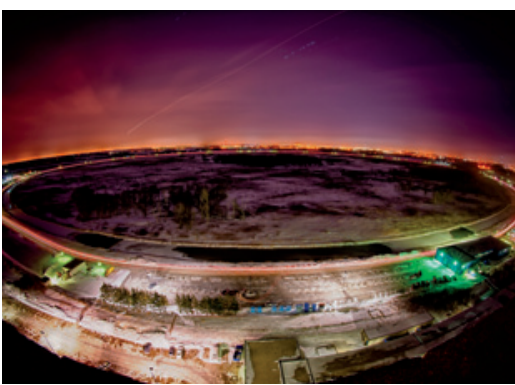

\section{JULY 1983}

Protons accelerated to a record 512 gigaelectronvolts.

\section{OCTOBER 1985}

Proton-antiproton collisions seen for the first time, at 1.6 teraelectronvolts.

\section{MARCH 1995}

The top quark discovered, the last fundamental constituent of matter. Its energy signatures are pictured, bottom.

\section{NOVEMBER 1996}

Observation of antihydrogen, the first antimatter atoms.

\section{MARCH 1998}

The $B_{c}$ meson, the last undiscovered quark-antiquark pair, is spotted.

\section{MARCH 2001}

Higher-energy phase of operation begins.

\section{SEPTEMBER 2006}

Discovery that $B_{s}$ mesons transform into their own antiparticles spontaneously.

\section{OCTOBER 2006}

Discovery of the $\Sigma^{ \pm}{ }_{b}$ baryons, which include a bottom quark.

\section{JUNE 2007}

Discovery of the $\Xi^{0}$ baryon, which includes a bottom and a strange quark.

\section{AUGUST 2008}

Experimental evidence restricts the possible mass range of the Higgs boson.
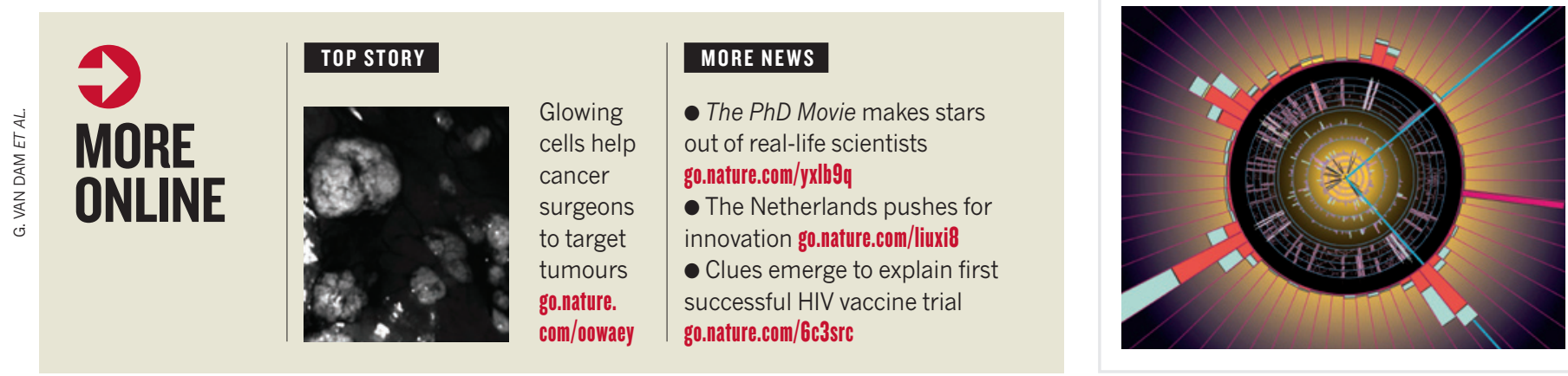